\title{
Are we less social in noisy environments? No effect of noise on implicit Theory of Mind performance
}

\author{
Louisa Kulke ${ }^{1 *}$, Hannes Rakoczy ${ }^{2}$ \\ ${ }^{1}$ Friedrich-Alexander-Universität Erlangen-Nürnberg \\ ${ }^{2}$ Georg-August-Universität Göttingen \\ *Corresponding author: Louisa Kulke, louisa.kulke@fau.de
}

Theory of Mind (ToM), the ability to attribute beliefs and desires to others, has been a recent focus of replication research. While some researchers found an implicit form of ToM, which could be measured with different tasks, including anticipatory looking measures, other researchers could not replicate these finding. The testing conditions may play a role for the success of replications. Therefore, the current study aimed to investigate the effect of a noisy testing environment on results in an anticipatory looking false belief task. The original findings could only be partially replicated, leaving room for alternative explanations. Environmental noise did not significantly affect gaze patterns. Therefore, previous failed replications are unlikely to be related to different levels in environmental noise.

\section{Introduction}

Theory of Mind (ToM), the ability to attribute beliefs, desires and intentions to others, plays an important role for social interactions in everyday life. Traditionally, this skill has been measured in verbal tasks, in which vignettes are told to participants, who then have to predict the outcome based on the protagonist's belief (Baron-Cohen, Leslie, \& Frith, 1985; Wimmer \& Perner, 1983). However, more recent paradigms claimed that it is possible to measure this skill implicitly, using eye-tracking (Clements \& Perner, 1994; Kovács, Téglás, \& Endress, 2010; Low \& Watts, 2013; Onishi \& Baillargeon, 2005; e.g. Schneider, Bayliss, Becker, \& Dux, 2012; Senju, Southgate, White, \& Frith, 2009; Southgate, Senju, \& Csibra, 2007; Surian \& Geraci, 2012), behavioural measures (e.g. D. Buttelmann, Carpenter, \& Tomasello, 2009; F. Buttelmann, Suhrke, \& Buttelmann, 2015; Fizke, Butterfill, Van de Loo, Reindl, \& Rakoczy, 2014; Knudsen \& Liszkowski, 2012; Southgate, Chevallier, \& Csibra, 2010) or other implicit tasks (Furlanetto, Becchio, Samson, \& Apperly, 2015;
Samson, Apperly, Braithwaite, Andrews, \& Bodley Scott, 2010; van der Wel, Sebanz, \& Knoblich, 2014). There is currently a debate whether these tasks reliably measure true mindreading skills, or whether the tasks are not sufficiently reliable for testing ToM as they are prone to alternative explanations (Baillargeon, Buttelmann, \& Southgate, 2018; Poulin-Dubois et al., 2018). In particular, several replications of original implicit ToM paradigms failed (Kulke \& Rakoczy, 2018). Even anticipatory looking (AL) tasks, which are automatized using eye-tracking and therefore less dependent on the experimenter, could often not be replicated (e.g. Grosse Wiesmann, Friederici, Disla, Steinbeis, \& Singer, 2018; Kulke, Johannsen, \& Rakoczy, 2019; Kulke \& Rakoczy, 2019; Kulke, Reiß, Krist, \& Rakoczy, 2017, 2018; Kulke, von Duhn, Schneider, \& Rakoczy, 2018; Kulke, Wübker, \& Rakoczy, 2019; Schuwerk, Vuori, \& Sodian, 2015).

This leaves room for two broad explanations. Firstly, findings from original studies may reflect artefacts due to lowlevel confounds. Secondly, external 
conditions may affect the (fragile) effects, leading to the failure to replicate findings. There are numerous possibilities for external interferences, including visual features (Kulke, Duhn, Schneider, \& Rakoczy, 2018), conditioning (Kulke, Duhn, et al., 2018) and the testing environment. As implicit ToM is impaired when subjects complete an additional task straining their attention (Schneider, Lam, Bayliss, \& Dux, 2012), distracting factors may affect belief-congruent gaze patterns. One factor that can be identified as highly distracting is environmental noise. Particularly, as developmental studies often take place in kindergartens with a certain level of environmental noise, this factor may affect findings. Furthermore, the volume level of stimulus videos used for implicit ToM tasks has recently been discussed as a factor influencing (non)replication. Relative volume depends on environmental noise, suggesting that this may be a relevant and related factor.

Therefore, the current study aimed to investigate the effect of natural environmental noise on performance in AL implicit ToM tasks. For this purpose, testing was conducted at a local family fair, simulating noisy environments often encountered in kindergartens. Either subjects were exposed to this noise, or they were wearing noise-cancelling headphones, filtering out this noise. It was predicted that if environmental noise plays a crucial role for replicability of the tasks, the paradigms should replicate if subjects wear noisecancelling headphones but not, or to a lesser degree, if they are exposed to environmental noise.

\section{Method}

\section{Participants}

The current study was preregistered with the Open Science Framework (https://osf.io/ca682/). Subject numbers were pre-determined as 56 based on the original studies. Healthy adult subjects between 18 and 60 years of age were recruited at a local family fair.

The current study used identical stimuli to Senju et al. (2009) and therefore, sample sizes were computed based on the effects found in the original study. To predict sample sizes, a normal distribution of the data was assumed and calculations were based on the formula provided by Rosner (2000):

$n \geq \frac{\left(F_{N(0,1)}^{-1}(1-\alpha)+F_{N(0,1)}^{-1}(1-\beta)\right)^{2} \cdot s d^{2}}{\left(m_{0}-m\right)^{2}}$

With sd being the (estimated) standard deviation of the data based on the previous studies, $m_{0}-m$ being the difference between the expected mean under the Null hypothesis and the mean measured in previous studies, $F_{N(0,1)}^{-1}(0.95)=1.645$ being the $95 \%$ quantile of the normal distribution and $F_{N(0,1)}^{-1}(0.8)=0.842$ the $80 \%$ quantile. Senju et al. (2009) report a differential looking score of $\mathrm{m}=0.42$ that is significantly different from $0(\mathrm{t}=2.76)$. The standard deviation can be calculated from the reported $\mathrm{t}, \mathrm{m}$ and $\mathrm{n}$ values as $s d=$ $\frac{m \cdot \sqrt{n}}{t}=\frac{0.42 \cdot \sqrt{17}}{2.76}=0.627$. Using the original mean, the minimum sample size needs to be $\mathrm{n}^{*} \geq 13.796 .{ }^{1}$ Four different conditions will be tested based on the independent variable stimulus video (FB1 and FB2, based on the original study) and noise level (high or

\footnotetext{
${ }^{1}$ Note however, that the original study uses one additional outcome measure (first saccade) for which, according to power calculations, a larger sample size would be required ( 25 subjects). However, the current study collected data at a family fair to simulate a noisy environment and this fair only takes place for 2 days, so that the time for data collection is limited. Therefore, only the outcome measure that requires a smaller sample size was analysed.
} 
low), leading to a total number of 56 subjects.

The study was approved by the university of Göttingen ethics committee (Ref. number: 143b).

\section{Apparatus and materials}

The current study includes exact replications of the methods described in the original paper, using original stimuli, shared with us by the authors. In this paradigm, an actress is watching two boxes from behind an occluder containing two windows. In the first two familiarisation trials, a toy is lying on top of one of the two boxes (first trial left, second trial right box), a chime sounds and the windows light up, followed by the actress reaching through the correct window to retrieve the toy. In the subsequent two familiarisation trials, a bear hand puppet appears with a ball, puts it in one of the two boxes (first trial left, second trial right box) and disappears. The chime sounds and the windows light up, followed by the actress retrieving the ball from the box. In the subsequent test trial (FB1), the bear puts the ball in the left box, then moves the ball from the left to the right box and disappears. A telephone sounds, the actress turns away, while the bear reappears and removes the ball from the scene. This test trial is counterbalanced with a second version (FB2), in which the bear puts the ball in the left box and disappears, a telephone sounds, the actress turns away, the bear returns, moves the ball to the other box and then retrieves it again to remove it from the scene. At the end of both trials, the actress turns back, the chime sounds and the windows light up. The direction of the first saccade after the chime and the looking time to both windows over the subsequent 6 seconds are measured. A differential looking score is calculated.

\section{Equipment}

An SMI RED250mobile eye tracker recorded gaze positions at a rate of $60 \mathrm{~Hz}$.
Before recording started, subjects completed a standard 5-point calibration and validation routine. Movies were controlled in the SMI Experiment Center (version 3.6.54) and presented on a 15" LCD screen (1920 x 1080 pixel). Gaze information was saved for offline analysis, which was conducted using BeGaze Software (version 3.6.52), R Studio and IBM SPSS Statistics (version 22).

\section{Procedure}

The testing took place at a family fair with a noisy environment. Noise measurements were taken at five different times on both days for 30 consecutive seconds. They showed an average background noise of $67.1 \mathrm{~dB}(S D=1.908$ $\mathrm{dB})$ with the maximum being $84.4 \mathrm{~dB}(S D$ $=3.323 \mathrm{~dB}$ ). To avoid visual impressions affecting the performance, subjects were facing the corner of a booth with white walls, so that no movements could be detected in their peripheral visual field. As the testing took place in a hall with no natural lighting, artificial lighting conditions were kept constant. Subjects signed an informed consent form and were seated in front of the eye-tracker. After the calibration procedure they were either instructed to put on noise cancelling headphones (headphones condition) or not (no headphones condition). They then viewed one of the stimulus videos (FB1 or FB2). After the testing process, they provided demographic details.

\section{Eye tracking analysis}

Looking times to the areas of interest over the two windows within the $6 \mathrm{~s}$ after illumination of the windows were determined as described in the original paper by Senju et al. (2009). The differential looking score (DLS) was calculated as the difference in looking time to the incorrect side AOI from the correct side AOI divided by the sum of looking times to both sides. 


$$
\text { looking score }=\frac{\mathrm{t}(\text { corr })-\mathrm{t}(\text { incorr })}{\mathrm{t}(\text { corr })+\mathrm{t}(\text { incorr })}
$$

T-tests were used to investigate whether the differential looking score differs from 0 in each condition (separately for high and low noise conditions and separately for the FB1 and FB2 condition of the original paradigm). Planned t-tests were conducted to compare the DLS between conditions.

Follow up Bayesian analyses were conducted using the "BayesFactor" Package (Morey \& Rouder, 2015) in R (Core Team, 2012) using Cauchy priors based on Liang, Paulo, Molina, Clyde, and Berger (2012).

\section{Exclusion}

It was originally planned (and preregistered) to exclude participants from the analysis if they do not show anticipatory looking to the correct location in the last familiarization trial as defined in the original study by Senju et al. (2009). However, due to the high number of subjects failing the inclusion criteria (see also Dörrenberg, Liszkowski, \& Rakoczy, 2018; Grosse Wiesmann et al., 2018; Kulke, Johannsen, et al., 2019; Kulke, Reiß, et al., 2018; Kulke, von Duhn, et al., 2018; Kulke, Wübker, et al., 2019) and the limited time to test under identical conditions, as the fair was only in town for 2 days, the exclusion criteria were not applied. However, a comparison of subject passing and failing the inclusion criteria shows that there was no significant difference in DLS between the groups.

\section{Results}

On the whole 58 subjects were tested, of which 2 had to be excluded because the calibration was unsuccessful ( $n$ $=1$ ) or because they were over the age of 60 $(n=1)$, leaving a total of 56 subjects in the sample. Bayes Factors were calculated in $\mathrm{R}$ using the BayesFactor package. T-tests were used to investigate whether the differential looking score differed from 0 in each condition (separately for high and low noise conditions and separately for the FB1 and FB2 condition of the original paradigm).

Figure 1 displays mean DLS as a function of conditions. DLS was positive, but not significantly positive in the FB1 condition with headphones $(M(14)=0.364$, $S D=0.756), t(13)=1.800, p=.095, B F_{10}=$ 1.000 , and the condition without headphones $(M(14)=0.331, S D=0.670)$, $t(13)=1.846, \mathrm{p}=.088, B F_{10}=1.029$. In the FB2 condition, DLS did not significantly differ from zero in the condition with headphones $(M(14)=0.086, S D=0.775)$, $t(13)=0.416, p=.684, B F_{10}=0.291$, or without headphones $(M(13)=-0.028, S D=$ $0.640), t(12)=-0.158, p=.877, B F_{10}=$ 0.308 .

Planned t-tests were conducted to compare DLS between conditions. Overall DLS did not significantly differ between the FB1 $(M(28)=0.347, S D=0.701)$ and the FB2 condition $(M(27)=0.031, S D=0.702)$, $t(53)=1.670, p=.101, B F_{10}=1.096$. There was no significant difference between subjects wearing no headphones $(M(27)=$ $0.158, S D=0.668)$ and subjects wearing headphones $(M(28)=0.225, S D=0.764)$, $t(53)=-0.345, p=.731, B F_{10}=0.303$.

When considering the FB1 and FB2 condition separately, there was no difference in DLS between subjects wearing headphones and subjects not wearing headphones in the FB1, $t(26)=$ $0.122, p=.904, B F_{10}=0.349$, or in the FB2 condition, $t(25)=-0.416, p=681, B F_{10}=$ 0.418 .

The difference between FB1 and FB2 conditions was not significant in the group not wearing headphones, $t(25)=$ $1.420, p=.168, B F_{10}=1.007$, or the group wearing headphones, $t(26)=0.959, p=$ $.346, B F_{10}=0.483$. 


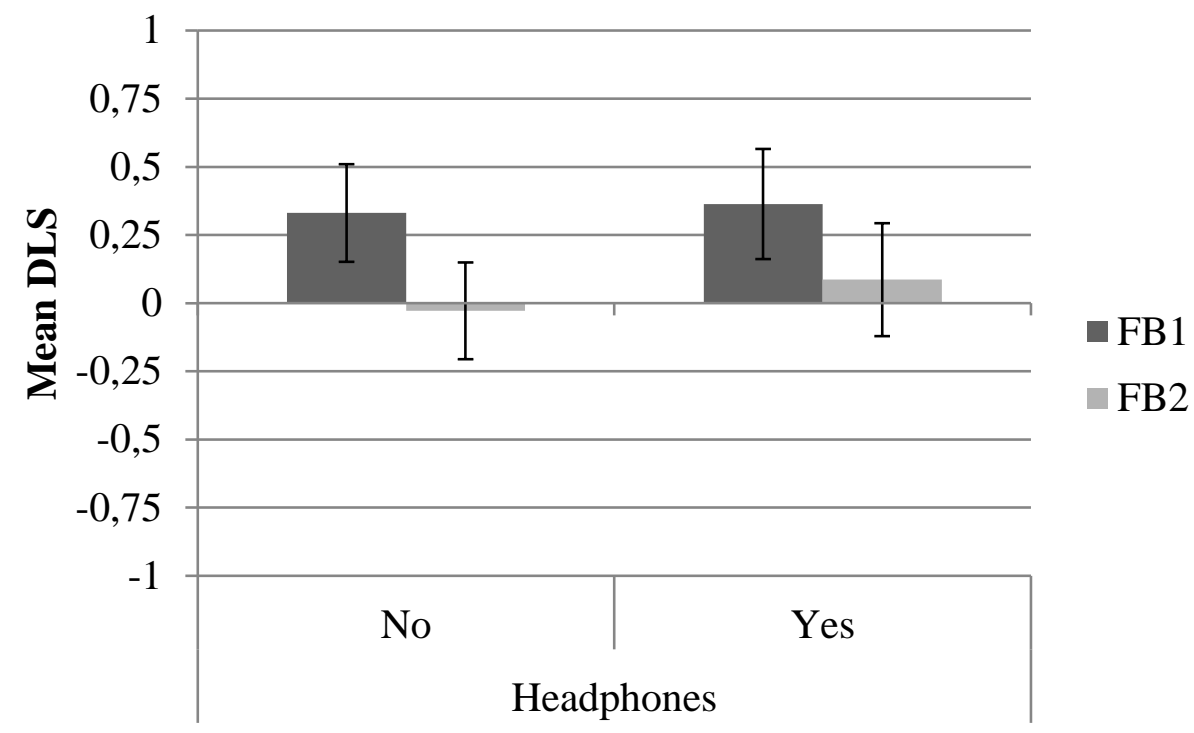

Figure 1. Mean DLS as a function of Condition (FB1/FB2) and Wearing of headphones (yes or no).

\section{Discussion}

The aim of the current study was to investigate the effect of environmental noise on belief-congruent looking patterns in implicit ToM tasks. Results show that belief-congruent looking patterns can only be detected in the FB1 condition, in which the belief-congruent location is identical to the last object location. If the last object location differs from the belief-congruent location, subjects do not preferably look at either location. The pattern was unaffected by the noise level subjects were exposed to, which was confirmed by Bayesian statistics.

The findings suggest that it is unlikely that environmental noise plays a role for the replicability of the AL paradigms, as similar patterns of results have been described using the original stimuli in quiet test rooms (e.g., Kulke, Duhn, et al., 2018). The present findings are in line with the growing body of nonreplications (e.g. Grosse Wiesmann et al., 2018; Kulke, Johannsen, et al., 2019; Kulke \& Rakoczy, 2019; Kulke et al., 2017; Kulke, Reiß, et al., 2018; Kulke, von Duhn, et al., 2018; Kulke, Wübker, et al., 2019; Schuwerk et al., 2015). They contrast original findings by Southgate et al. (2007) and Senju et al. (2009). The current study suggests that environmental noise is unlikely to affect the replicability of $\mathrm{AL}$ ToM tasks.

As the study took place at a fair that was only in town for a limited time, only a limited number of subjects could be tested. The predetermined number of subjects based on the power of original studies was reached. However, not all of the participants passed the original inclusion criteria based on looking patterns during familiarisation trials. This could have led to insufficient sample sizes to detect effects. However, the current analyses, as well as previous literature (Kulke et al., 2017; Kulke, Reiß, et al., 2018) suggest that there is no difference in gaze pattern between subjects passing and failing the familiarisation procedure, suggesting that the inclusion of these participants should not distort findings.

In conclusion, the current study found gaze patterns in an anticipatory looking Theory of Mind task to be independent of environmental noise levels. 


\section{References}

Baillargeon, R., Buttelmann, D., \& Southgate, V. (2018). Interpreting failed replications of early falsebelief findings: Methodological and theoretical considerations. Cognitive Development.

Baron-Cohen, S., Leslie, A. M., \& Frith, U. (1985). Does the autistic child have a "theory of mind"? Cognition, 21(1), 37-46.

Buttelmann, D., Carpenter, M., \& Tomasello, M. (2009). Eighteen-month-old infants show false belief understanding in an active helping paradigm. Cognition, 112(2), 337-342.

Buttelmann, F., Suhrke, J., \& Buttelmann, D. (2015). What you get is what you believe: Eighteenmonth-olds demonstrate belief understanding in an unexpected-identity task. Journal of experimental child psychology, 131, 94-103.

Clements, W. A., \& Perner, J. (1994). Implicit understanding of belief. Cognitive Development, 9(4), 377-395.

Core Team, R. (2012). R: A language and environment for statistical computing. Vienna, Austria.

Dörrenberg, S., Liszkowski, U., \& Rakoczy, H. (2018). How (not) to measure infant Theory of Mind: Testing the replicability and validity of four non-verbal measures. Cognitive Development.

Fizke, E., Butterfill, S., Van de Loo, L., Reindl, E., \& Rakoczy, H. (2014). Signature limits in early theory of mind: Toddlers spontaneously take into account false beliefs about an object's location but not about its identity. Department of Psychology, University of Göttingen.

Furlanetto, T., Becchio, C., Samson, D., \& Apperly, I. (2015). Altercentric Interference in Level 1 Visual Perspective Taking Reflects the Ascription of Mental States, Not Submentalizing.

Grosse Wiesmann, C., Friederici, A. D., Disla, D., Steinbeis, N., \& Singer, T. (2018). Longitudinal evidence for 4-year-olds' but not 2- and 3-year-olds' false belief-related action anticipation. Cognitive Development 46, 58-68. doi:https://doi.org/10.1016/j.cogdev.2017.08.007

Knudsen, B., \& Liszkowski, U. (2012). 18-month-olds predict specific action mistakes through attribution of false belief, not ignorance, and intervene accordingly. Infancy, 17(6), 672-691.

Kovács, Á. M., Téglás, E., \& Endress, A. D. (2010). The social sense: Susceptibility to others' beliefs in human infants and adults. science, 330(6012), 1830-1834.

Kulke, L., Duhn, B. v., Schneider, D., \& Rakoczy, H. (2018). Is Implicit Theory of Mind a Real and Robust Phenomenon? Results From a Systematic Replication Study. Psychological Science, 29(6), 888--900. doi:10.1177/0956797617747090

Kulke, L., Johannsen, J., \& Rakoczy, H. (2019). Why can some implicit Theory of Mind tasks be replicated and others cannot? A test of mentalizing versus submentalizing accounts. PloS one, 14(3), e0213772.

Kulke, L., \& Rakoczy, H. (2018). Implicit Theory of Mind-An overview of current replications and non-replications. Data in Brief, 16, 101-104.

Kulke, L., \& Rakoczy, H. (2019). Testing the Role of Verbal Narration in Implicit Theory of Mind Tasks. Journal of Cognition and Development, 20(1), 1-14. doi:10.1080/15248372.2018.1544140

Kulke, L., Reiß, M., Krist, H., \& Rakoczy, H. (2017). Implicit Theory of Mind across the life span Anticipatory looking data. Data in Brief, 15 (Supplement C), 712-719. doi:https://doi.org/10.1016/j.dib.2017.10.021

Kulke, L., Reiß, M., Krist, H., \& Rakoczy, H. (2018). How robust are anticipatory looking measures of Theory of Mind? Replication attempts across the life span. Cognitive Development, 46, 97-111. doi:https://doi.org/10.1016/j.cogdev.2017.09.001

Kulke, L., von Duhn, B., Schneider, D., \& Rakoczy, H. (2018). Is Implicit Theory of Mind a Real and Robust Phenomenon? Results From a Systematic Replication Study. Psychological Science, $O(0), 0956797617747090$. doi:10.1177/0956797617747090

Kulke, L., Wübker, M., \& Rakoczy, H. (2019). Is implicit Theory of Mind real but hard to detect? Testing adults with different stimulus materials. Royal Society Open Science, 6(7), 190068.

Liang, F., Paulo, R., Molina, G., Clyde, M. A., \& Berger, J. O. (2012). Mixtures of g priors for Bayesian variable selection. Journal of the American Statistical Association.

Low, J., \& Watts, J. (2013). Attributing false beliefs about object identity reveals a signature blind spot in humans' efficient mind-reading system. Psychological Science, 24(3), 305-311. 
Morey, R. D., \& Rouder, J. N. (Producer). (2015). BayesFactor: Computation of Bayes Factors for Common Designs. $R$ package version 0.9.12-2. Retrieved from http://CRAN.Rproject.org/package $=$ BayesFactor

Onishi, K. H., \& Baillargeon, R. (2005). Do 15-month-old infants understand false beliefs? science, 308(5719), 255-258.

Poulin-Dubois, D., Rakoczy, H., Burnside, K., Crivello, C., Dörrenberg, S., Edwards, K., . . Low, J. (2018). Do infants understand false beliefs? We don't know yet-A commentary on Baillargeon, Buttelmann and Southgate's commentary. Cognitive Development, 48, 302-315.

Samson, D., Apperly, I. A., Braithwaite, J. J., Andrews, B. J., \& Bodley Scott, S. E. (2010). Seeing it their way: evidence for rapid and involuntary computation of what other people see. Journal of experimental psychology: human perception and performance, 36(5), 1255.

Schneider, D., Bayliss, A. P., Becker, S. I., \& Dux, P. E. (2012). Eye movements reveal sustained implicit processing of others' mental states. Journal of experimental psychology: general, 141(3), 433.

Schneider, D., Lam, R., Bayliss, A. P., \& Dux, P. E. (2012). Cognitive load disrupts implicit theory-ofmind processing. Psychological Science, 0956797612439070.

Schuwerk, T., Vuori, M., \& Sodian, B. (2015). Implicit and explicit theory of mind reasoning in autism spectrum disorders: the impact of experience. Autism, 19(4), 459-468.

Senju, A., Southgate, V., White, S., \& Frith, U. (2009). Mindblind eyes: an absence of spontaneous theory of mind in Asperger syndrome. science, 325(5942), 883-885.

Southgate, V., Chevallier, C., \& Csibra, G. (2010). Seventeen-month-olds appeal to false beliefs to interpret others' referential communication. Developmental Science, 13(6), 907-912.

Southgate, V., Senju, A., \& Csibra, G. (2007). Action anticipation through attribution of false belief by 2-year-olds. Psychological Science, 18(7), 587-592.

Surian, L., \& Geraci, A. (2012). Where will the triangle look for it? Attributing false beliefs to a geometric shape at 17 months. British Journal of Developmental Psychology, 30(1), 30-44.

van der Wel, R. P., Sebanz, N., \& Knoblich, G. (2014). Do people automatically track others' beliefs? Evidence from a continuous measure. Cognition, 130(1), 128-133.

Wimmer, H., \& Perner, J. (1983). Beliefs about beliefs: Representation and constraining function of wrong beliefs in young children's understanding of deception. Cognition, 13(1), 103-128. 